\title{
String tensions and deconfinement transition in the SU(4) center vortex model
}

\section{Michael Engelhardt ${ }^{* \dagger}$}

New Mexico State University

E-mail: engel@nmsu.edu

\begin{abstract}
A center vortex model for the infrared sector of $S U$ (4) Yang-Mills theory is constructed such as to reproduce both the ratio between the zero-temperature quark and diquark string tensions known from lattice Yang-Mills theory, as well as the properties of the deconfinement transition. On this basis, the temperature dependence of the spatial quark and diquark string tensions is predicted. Though still phenomenologically viable, details of the construction of the $S U(4)$ center vortex model corroborate previous arguments that modeling infrared Yang-Mills dynamics purely in terms of vortex world-surface characteristics may become less appropriate as the number of colors is increased.
\end{abstract}

XXIIIrd International Symposium on Lattice Field Theory

25-30 July 2005

Trinity College, Dublin, Ireland

*Speaker.

${ }^{\dagger}$ Supported by the U.S. DOE under grant number DE-FG03-95ER40965. 


\section{Introduction and Motivation}

Random vortex world-surface models are designed to describe the infrared sectors of $S U(N)$ Yang-Mills theories. They are based on the notion that the relevant infrared degrees of freedom are center vortices, i.e., closed tubes of quantized chromomagnetic flux $[1,2,3,4,5,6]$. In practice, ensembles of center vortex world-surface configurations are generated using Monte Carlo methods, where the world-surfaces are composed of elementary squares on a hypercubic lattice.

In the case of $S U(2)$ color, such a vortex model has proven to furnish a comprehensive description of the central mechanisms governing the strong interaction in the infrared [7, 8, 9]. Both the confined and deconfined phases of the corresponding Yang-Mills theory are generated, separated by a second order finite-temperature deconfinement transition. In addition to the confining properties, also the topological properties encoded in the topological susceptibility are reproduced quantitatively; furthermore, the (quenched) chiral condensate in the vortex ensemble is quantitatively compatible with the behavior found in $S U$ (2) Yang-Mills theory.

In the $S U(3)$ case, the random vortex world-surface model shows similar promise $[10,11$, 12]. While the topological properties and the coupling to quark degrees of freedom remain to be investigated, the $S U(3)$ vortex model again generates both the confined and deconfined phases, separated by a weak first order deconfinement transition. The static baryonic potential satisfies a $Y$ law and the vortex free energy displays the correct behavior as a dual order parameter.

In both the $S U(2)$ and $S U(3)$ models, only one dimensionless parameter characterizing the vortex world-surface dynamics needs to be adjusted to arrive at the quantitative agreement with lattice Yang-Mills theory highlighted above. This parameter controls vortex stiffness, i.e., it is the coefficient of a curvature action term. On the other hand, this simple picture, with dynamics controlled purely by one vortex world-surface characteristic, is not expected to persist for a larger number of colors, as was previously argued in [13]. This can be understood as follows: Generic vortex world-surfaces are not orientable, which implies that they cannot be described by a field strength which points into a single direction in color space. Rather, the color direction of the field strength must have some amount of variation as one varies position on the vortex, in any gauge. In particular, for many purposes it is convenient to construct the vortex field strength in an Abelian fashion, such that it is restricted to a definite (Cartan) color component for each possible type of vortex flux. In this case, the field strength in general is forced to jump at certain lines defined on the vortex world-surface in a way which corresponds to having a source or sink of magnetic flux at those lines. In other words, these lines constitute the world-lines of Abelian monopoles, which are thus an intrinsic feature of generic, non-orientable, vortex world-surface configurations in Abelian gauges $[14,15,8]$. Moreover, in the case of three or more colors, these monopoles appear not only as locations at which the field strength on a vortex jumps, but the vortex flux may also branch there; in general, for $N$ colors, up to $N$ center vortex fluxes can emanate from any given monopole.

As the number of colors $N$ rises, center flux can be quantized in ever smaller units, while the monopoles always constitute sources or sinks of the same total amount of flux, cf. Fig. 1. It seems plausible that this may lead to a shift in the relative importance of pure vortex world-surface characteristics and monopole characteristics for the dynamics of the vortex ensemble. While at low $N$, the monopole dynamics appear to be completely determined by the dynamics of the vortices on which the monopoles reside, at higher $N$, the monopoles may attain their own dynamic signif- 

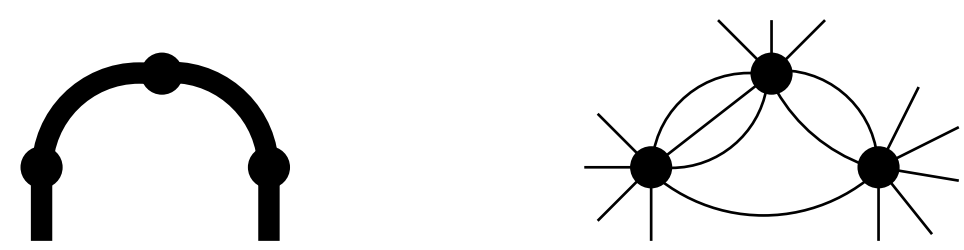

Figure 1: Vortex configurations contain monopoles. For $N$ colors, up to $N$ vortex fluxes can emanate from any given monopole. Examples for $N=2$ (left) and $N=8$ (right) are depicted. As $N$ rises, center flux can be quantized in smaller units, while monopoles remain sources or sinks of the same total amount of flux.

icance and may influence the overall dynamics of the vortex world-surfaces. Thus, a pure vortex world-surface curvature action, which appears to be entirely sufficient to model $S U(2)$ and $S U(3)$ Yang-Mills theory in the infrared regime, may well cease to be appropriate at higher $N$. Definite indications of this occur in the $S U(4)$ case investigated in the present work, which thus aims to probe the limits of applicability of a pure random world-surface dynamics for center vortices.

\section{SU(4) vortex model}

The $S U$ (4) gauge group contains the four center elements $\{1, i,-1,-i\}$, which determine the quantization of vortex flux; vortices are characterized by contributing a center phase to any Wilson loop to which they are linked. The trivial unit center element corresponds to no vortex flux being present, and vortices generating the phases $i$ and $-i$ are related by a space-time inversion. Therefore, there are actually two different types of vortices; the ones generating a -1 phase and the ones generating the phases $\pm i$ depending on their orientation in space-time.

Correspondingly, $S U$ (4) Yang-Mills theory contains two different string tensions, the quark string tension $\sigma_{1}$ and the diquark string tension $\sigma_{2}$. Contrary to the $S U(2)$ and $S U(3)$ cases, where one string tension measurement is needed as input to fix the stiffness of one vortex type, for $S U(4)$, measurements of both string tensions, $\sigma_{1}$ and $\sigma_{2}$, are needed as input to fix the stiffness of the two vortex types in $S U(4)$. In practice, the two pieces of data from lattice Yang-Mills "experiment" used to fix the curvature action are ( $T_{C}$ denoting the deconfinement temperature) $[16,17]$

$$
\sigma_{2}(T=0) / \sigma_{1}(T=0)=1.36 \quad T_{C} / \sqrt{\sigma_{1}(T=0)}=0.62
$$

A $S U$ (4) vortex action using two stiffness parameters for the two types of vortices would constitute the direct generalization of the actions used in the $S U(2)$ and $S U(3)$ cases. However, initial investigation of such a model, adjusting two stiffness parameters to the inputs (2.1), led to the conclusion that it is not phenomenologically viable. Specifically, the deconfinement transition, if at all first order, turned out to have an undetectably low latent heat, certainly considerably lower than found in the $S U(3)$ vortex model. This is in qualitative disagreement with $S U(4)$ lattice Yang-Mills theory, where the transition is roughly twice as strongly first order [16] as for $S U(3)$. Thus, as detailed below, an additional action term favoring vortex branching was introduced, and its strength was fixed using a third input from lattice Yang-Mills "experiment", characterizing the deconfinement transition [16]:

$$
\left.\Delta S \frac{T_{C}^{4}}{\sigma_{1}^{4}(T=0)}\right|_{S U(4)}=\left.2 \cdot \Delta S \frac{T_{C}^{4}}{\sigma^{4}(T=0)}\right|_{S U(3)}
$$


where $\Delta S$ denotes the latent heat. In practice, the center vortex world-surfaces are modeled by composing them of elementary squares on a hypercubic lattice. The lattice spacing in this approach is a fixed physical quantity implementing the notion that vortices possess a finite transverse thickness and must be a minimal distance apart to be distinguished from one another. Vortex world-surfaces on lattices are generated using Monte Carlo methods. The action can be represented as

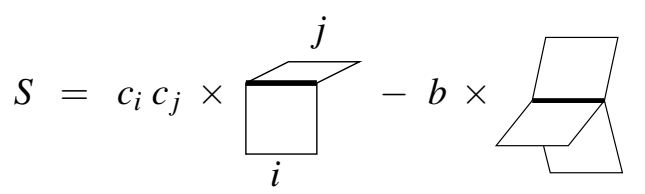

The first term is the curvature term; it penalizes configurations in which two vortex squares share a link without lying in the same plane. This directly generalizes the action used in the $S U(2)$ and $S U(3)$ cases. There, only one type of vortex exists, and there is only one curvature coefficient weighting vortex world-surfaces "going around a corner". In the present case, the strength of the curvature action varies according to the different possible types of vortex squares meeting at a link. Note that it would be quite admissible to use a more general curvature coefficient $c_{i j}$ with $c_{12} \neq c_{1} c_{2}$. This option was explored, but did not seem to enhance the phenomenological flexibility of the model. The same is true for an area action term which simply penalizes the total vortex surface area, the effects of which can in practice be absorbed into the curvature term [7].

The second term in the action, on the other hand, encourages vortex branching: The action is decremented by the branching coefficient $b$ for each link which has 3 or 5 vortex squares attached to it. This happens precisely when a vortex branches. As indicated above, this term is introduced in order to enhance the first order character of the deconfinement transition. The underlying rationale is this: The $S U(2)$ and $S U(3)$ models are governed by the same type of action; they differ in the topology of the configurations. Only the $S U(3)$ model contains vortex branchings and only it exhibits a first order deconfinement transition. Thus, it appears plausible that enhancing branchings in the $S U$ (4) model may restore a detectable first order deconfinement transition of the correct strength, cf. (2.2). This is indeed confirmed by the measurements described below. Before proceeding to discuss these, it should be noted that vortex branchings are associated with the presence of a monopole if one specifies the vortex field strength such that it is restricted to a definite (Cartan) color component for each type of vortex flux. Therefore, the branching coefficient $b$ can also be interpreted as favoring the presence of monopoles. In this sense, the action (2.3) accords monopoles their own dynamical significance and ceases to rely purely on vortex world-surface characteristics.

In practice, the physical point of the model was found as follows. Since only discrete temperatures can be realized using a fixed lattice spacing, at the physical point in parameter space the deconfinement transition is in general not directly accessible. Instead, the physical point is fixed by interpolation of parameter sets at which an integer multiple of the lattice spacing indeed corresponds to the inverse deconfinement temperature. In practice, measurements were carried out on lattices with $N_{t}=1,2,3$ spacings in the temporal direction, and the coupling constants $c_{1}, c_{2}$ and $b$ were fixed such that the deconfinement temperature is realized, with the first condition in (2.1) and the condition (2.2) satisfied as well. The result is displayed in Table 1, along with the ratio $T_{C} / \sqrt{\sigma_{1}}$ obtained at each parameter set. The physical point is found from these data by interpolating quadratic functions of $T_{C} / \sqrt{\sigma_{1}}$ for the three coupling constants. Setting $T_{C} / \sqrt{\sigma_{1}}=0.62$, which is the final condition to be satisfied at the physical point, cf. (2.1), defines the physical parameters 


$$
c_{1}=0.45 \quad c_{2}=0.80 \quad b=0.71
$$

As is evident from Table 1, the physical point is very near the parameters obtained for $N_{t}=2$; the uncertainties inherent in the interpolation thus remain small. As a cross-check, it was verified that the first condition in (2.1) remains satisfied for the parameters (2.4).

\begin{tabular}{|c||c|c|c||c|}
\hline & $c_{1}$ & $c_{2}$ & $b$ & $T_{C} / \sqrt{\sigma_{1}}$ \\
\hline \hline$N_{t}=1$ & 0.2785 & 0.4005 & 0.1403 & 0.90 \\
\hline$N_{t}=2$ & 0.4558 & 0.7983 & 0.6950 & 0.61 \\
\hline$N_{t}=3$ & 0.5925 & 0.7059 & 0.3800 & 0.50 \\
\hline
\end{tabular}

Table 1: Sets of coupling constants realizing the deconfinement temperature as well as satisfying two of the three conditions defining the physical point. The last condition, $T_{C} / \sqrt{\sigma_{1}}=0.62$, is satisfied by interpolating the parameters using the data in the final column.

Having found the physical point, the behavior of the string tensions as a function of temperature can be predicted. In particular, the spatial string tensions $\sigma_{1}^{S}$ and $\sigma_{2}^{S}$ in the deconfined phase are of interest. Using a lattice with $N_{t}=1$ realizes the temperature $T=1.94 T_{C}$, and one obtains $\sigma_{1}^{S}\left(T=1.94 T_{C}\right)=1.34 \sigma_{1}(T=0)$ and $\sigma_{2}^{S}\left(T=1.94 T_{C}\right)=1.44 \sigma_{2}(T=0)$. The characteristic rise of the spatial string tensions in the deconfined phase is observed; more specifically, this rise is such that the ratio $\sigma_{2}^{S} / \sigma_{1}^{S}\left(T=1.94 T_{C}\right)=1.46$ is enhanced compared to the zero-temperature value 1.36 . For comparison, if one considers the simplified model with no branching term, $b=0$, and in turn disregards condition (2.2), this ratio takes the value 1.25 , smaller than the zero-temperature value.

\section{Conclusions}

A description of the infrared sector of $S U$ (4) Yang-Mills theory in terms of a random vortex world-surface ensemble is viable; however, the effortless predictivity of the $S U(2)$ and $S U(3)$ models is lost. In those cases, it proved sufficient to base the dynamics on a single vortex worldsurface characteristic, namely the curvature. By contrast, in the $S U(4)$ case, it is not only necessary to introduce two different curvature coefficients due to the existence of two different vortex types; in addition, a description based purely on world-surface properties had to be abandoned in favor of an action which also endows monopoles (which are intrinsically present in vortex configurations) with their own dynamical significance. This corroborates related arguments put forward in [13].

Three dimensionless parameters had to be tuned in order to correctly reproduce the properties (2.1) and (2.2). The model thus seems rather less attractive in terms of predictive power than the $S U(2)$ and $S U$ (3) models. Nevertheless, the behavior of the spatial string tensions in the deconfined phase was predicted. During the conference, the author became aware of newer lattice Yang-Mills data to which these quantities can be compared [18]. According to these data, the ratio of the (spatial) diquark and quark string tensions remains very near its zero-temperature value $\sigma_{2} / \sigma_{1}=$ 1.36 at high temperatures. Comparing with the results quoted in the previous section, introducing the branching term into the action (2.3) thus shifts the value of $\sigma_{2} / \sigma_{1}$ at high temperatures in the correct direction compared with the $b=0$ model; however, the correction overshoots the lattice Yang-Mills data considerably. Presumably, also this could be adjusted, e.g., by introducing an 
additional free parameter $c_{12} \neq c_{1} \cdot c_{2}$ in place of the combination $c_{1} \cdot c_{2}$ into the action (2.3). Without going into further detail concerning such generalized models, this discussion does clearly underscore once more the observation made at the beginning of this section, that the effortless predictivity of the $S U(2)$ and $S U(3)$ cases is lost in the $S U(4)$ random vortex world-surface model.

\section{References}

[1] G.'t Hooft, On the phase transition towards permanent quark confinement, Nucl. Phys. B138 (1978) 1.

[2] G. Mack, Predictions of a theory of quark confinement, Phys. Rev. Lett. 45 (1980) 1378.

[3] H. B. Nielsen and P. Olesen, A quantum liquid model for the QCD vacuum: Gauge and rotational invariance of domained and quantized homogeneous color fields, Nucl. Phys. B160 (1979) 380.

[4] L. Del Debbio, M. Faber, J. Giedt, J. Greensite and Š. Olejník, Detection of center vortices in the lattice Yang-Mills vacuum, Phys. Rev. D 58 (1998) 094501 [hep-lat/9801027].

[5] M. Engelhardt, K. Langfeld, H. Reinhardt and O. Tennert, Deconfinement in SU(2) Yang-Mills theory as a center vortex percolation transition, Phys. Rev. D 61 (2000) 054504 [hep-lat/99040 04 ].

[6] P. de Forcrand and M. D'Elia, On the relevance of center vortices to QCD, Phys. Rev. Lett. 82 (1999) 4582 [hep-lat/9901020].

[7] M. Engelhardt and H. Reinhardt, Center vortex model for the infrared sector of Yang-Mills theory: Confinement and deconfinement, Nucl. Phys. $\mathbf{B 5 8 5}$ (2000) 591 [hep-lat/9912003].

[8] M. Engelhardt, Center vortex model for the infrared sector of Yang-Mills theory: Topological susceptibility, Nucl. Phys. B585 (2000) 614 [hep-lat / 0004013 ].

[9] M. Engelhardt, Center vortex model for the infrared sector of Yang-Mills theory: Quenched Dirac spectrum and chiral condensate, Nucl. Phys. B638 (2002) 81 [hep-lat/ 0204002 ].

[10] M. Engelhardt, M. Quandt and H. Reinhardt, Center vortex model for the infrared sector of SU(3) Yang-Mills theory: Confinement and deconfinement, Nucl. Phys. B685 (2004) 227 [hep-lat/0311029].

[11] M. Engelhardt, Center vortex model for the infrared sector of SU(3) Yang-Mills theory: Baryonic potential, Phys. Rev. D 70 (2004) 074004 [hep-lat/ 0406022 ].

[12] M. Quandt, H. Reinhardt and M. Engelhardt, Center vortex model for the infrared sector of SU(3) Yang-Mills theory: Vortex free energy, Phys. Rev. D 71 (2005) 054026 [hep-lat/ 0412033 ].

[13] J. Greensite and Š. Olejník, $k$-string tensions and center vortices at large N, JHEP 0209 (2002) 039 [hep-lat/0209088].

[14] J. M. Cornwall, Nexus solitons in the center vortex picture of QCD, Phys. Rev. D 58 (1998) 105028 [hep-th/9806007].

[15] M. Engelhardt and H. Reinhardt, Center projection vortices in continuum Yang-Mills theory, Nucl. Phys. B567 (2000) 249 [hep-th/9907139].

[16] B. Lucini, M. Teper and U. Wenger, The high temperature phase transition in $S U(N)$ gauge theories, JHEP 0401 (2004) 061 [hep-lat/ 0307017 ].

[17] B. Lucini, M. Teper and U. Wenger, Glueballs and k-strings in $S U(N)$ gauge theories: Calculations with improved operators, JHEP 0406 (2004) 012 [hep-lat / 040400 8].

[18] B. Lucini, M. Teper and U. Wenger, Properties of the deconfining phase transition in SU $(N)$ gauge theories, JHEP 0502 (2005) 033 [hep-lat/ 0502003 ]. 